\title{
DIFFEOMORPHISMS FOR HILBERT MANIFOLDS AND HANDLE DECOMPOSITION
}

\author{
BY DAN BURGHELEA ${ }^{1}$
}

Communicated by Richard Palais, June 6, 1969

1. We announce here the following result:

Two homotopic diffeomorphisms of a paracompact separable hilbert manifold of infinite dimension are isotopic.

(1) In this paper, a hilbert manifold ( $h$-manifold) with or without boundary is always hausdorff, paracompact, separable $C^{\infty}$-differentiable and with the infinite dimensional separable hilbert space $H$ as local model.

Let $M(M, \partial M)$ be an $h$-manifold (with boundary), $X(X, \partial X)$, an $h$-manifold or finite dimensional manifold (with boundary).

(a) A closed imbedding $\phi: X \rightarrow M(\phi:(X, \partial X) \rightarrow(M, \partial M))$ is a $C^{\infty}$-injective map $\phi: X \rightarrow M$, such that the differential $d_{*} \phi(x)$ is injective for any $x$, and $\phi(M)$ is closed (for the case with boundary we ask more, $\phi^{-1}(\partial M)=\partial M$ and $\phi$ is transversal to $\partial M$ in $\left.\partial M\right)$.

(b) A closed tubular neighborhood of a closed imbedding of infinite codimension, $\phi: X \rightarrow M,(\phi:(X, \partial X) \rightarrow(M, \partial M))$, is a closed imbedding $\tilde{\phi}: X \times D^{\infty} \rightarrow M\left(\tilde{\phi}:(X, \partial X) \times D^{\infty} \rightarrow(M, \partial M)\right)$ which extends to an open imbedding $\bar{\phi}: X \times H \rightarrow M(\bar{\phi}:(X, \partial X) \times H \rightarrow(M, \partial M)$ with $\left.\bar{\phi}^{-1}(\partial M)=\partial X \times H\right)$.

Remarks. (1) Any closed imbedding of infinite codimension has closed tubular neighborhoods [3].

(2) For $\tilde{\phi}_{1}$ and $\tilde{\phi}_{2}$ two closed tubular neighborhoods of a closed imbedding $\phi: X \rightarrow M(\phi:(X, \partial X) \rightarrow(M, \partial M))$, there exists an isotopy $h_{t}: M \rightarrow M, \quad\left(h_{t}:(M, \partial M) \rightarrow(M, \partial M)\right), 0 \leqq t \leqq 1$, such that $h_{0}=$ id, $h_{t} \cdot \phi=\phi$ and $h_{1} \cdot \tilde{\phi}_{1}=\tilde{\phi}_{2}[2$, Theorem 4.1]. By an isotopy as in [2], we mean a level preserving $C^{\infty}$-diffeomorphism $h: M \times I \rightarrow M \times I$, $(h:(M, \partial M) \times I \rightarrow(M, \partial M) \times I)$, i.e., $h(x, t)=\left(h_{t}(x), t\right)$.

(c) Let $M(M, \partial M)$ be an $h$-manifold (with boundary); $A$ closed imbedded submanifold with boundary $(A, \partial A)$, such that $A \subset$ Int $M$ and $A \backslash \partial A$ is open submanifold of $M$, is called a zero-codimensional closed submanifold (0-c-submanifold).

The 0-c-submanifold $(B, \partial B)$ is called a collar neighborhood of the $0-c$-submanifold $(A, \partial A)$, if $A \subset \operatorname{Int} B$ and ( $B \backslash \operatorname{Int} A, \partial(B \backslash \operatorname{Int} A))$ is diffeomorphic to $(\partial A \times[0,1], \partial A \times \partial[0,1])$.

1 The author was partially supported by the National Science Foundation. 
Remarks. (1) For any 0-c-submanifold $(A, \partial A)$ of $M(M, \partial M)$, there exist collar neighborhoods.

(2) Suppose $\left(A_{1}, \partial A_{1}\right),\left(A_{2}, \partial A_{2}\right),\left(A_{3}, \partial A_{3}\right), 0$-c-submanifolds of $M(M, \partial M)$, such that $\left(A_{i+1}, \partial A_{i+1}\right)$ is a collar neighborhood of $\left(A_{i}\right.$, $\left.\partial A_{i}\right)$. There exists an isotopy $h_{t}: M \rightarrow M\left(h_{t}:(M, \partial M) \rightarrow(M, \partial M)\right)$ such that $h_{0}=\mathrm{id},\left.h_{t}\right|_{A_{1}}=\mathrm{id}$ and $h_{1}\left(A_{2}\right)=A_{3}$.

(d) Let $(M, \partial M)$ be an $h$-manifold with boundary. A collar neighborhood of $\partial M$ is a closed submanifold $(V, \partial V)$ with $\partial V=\partial_{0} V \cup \partial_{1} V$ (disjoint union), $\partial_{0} V=\partial M, \partial_{1} V \subset$ Int $M$, Int $V$ open submanifold of Int $M$, such that there exists a diffeomorphism $\theta:(V, \partial V) \rightarrow$ $(\partial M \times[0,1], \partial M \times \partial[0,1])$.

REMARKs. (1) Given $(M, \partial M)$, there exist collar neighborhoods of $\partial M$.

(2) Supposing $\left(V_{1}, \partial V_{1}\right),\left(V_{2}, \partial V_{2}\right),\left(V_{3}, \partial V_{3}\right)$ such that $V_{i} \subset$ $V_{i+1} \backslash \partial_{1} V_{i+1}$, there exists an isotopy $h_{t}:(M, \partial M) \rightarrow(M, \partial M)$ with $h_{0}=\mathrm{id}, h_{t} \mid V_{1}=\mathrm{id}, h_{1}\left(V_{2}\right)=V_{3}$.

(e) Let $(M, \partial M)$ be an $h$-manifold with boundary $(X, \partial X)$ an $h$ manifold or a finite dimensional manifold with boundary, $\phi:(X, \partial X)$ $\rightarrow(M, \partial M)$ a closed imbedding of infinite codimension, $\tilde{\phi}$ a closed tubular neighborhood, $(V, \partial V)$ a collar neighborhood of $\partial M$, and $\theta:(V, \partial V) \rightarrow(\partial M \times[0,1], \partial M \times[0,1])$ a diffeomorphism.

(1) $\phi$ is called transversal to $(V, \theta)$ if $\theta$ is a diffeomorphism of pairs $(V, \phi(X) \cap V) \rightarrow\left(\partial_{0} M \times[0,1], \phi(\partial X) \times[0,1]\right)$.

(2) $\tilde{\phi}$ is called transversal to $(V, \theta)$, with respect to the exten$\operatorname{sion} \phi$, if $\theta$ is a diffeomorphism of pairs

$$
(V, \Phi(X \times H) \cap V \rightarrow(\partial M \times[0,1], \Phi(\partial X \times H) \times[0,1]) .
$$

If $\theta:(V, \partial V) \rightarrow(\partial M \times[0,1], \partial M \times \partial[0,1])$ is our collar neighborhood, for any $\alpha, 0<\alpha<1$ we denote by $\alpha V$ and $\alpha \theta, \theta^{-1}(\partial M \times[0, \alpha])$ respectively $\nu_{\alpha} \cdot \theta$, where $\nu_{\alpha}$ is the diffeomorphism $\nu_{\alpha}: \partial M \times[0, \alpha]$ $\rightarrow \partial M \times[0,1]$ defined by $\nu_{\alpha}(x, s)=(x, s / \alpha)$. For short $(\alpha V, \alpha \theta)$ will be denoted by $\alpha(V, \theta)$; it is a collar neighborhood of $\partial M$.

Proposition 1.1. If $(M, \partial M)$ is an h-manifold with boundary, $(X, \partial X)$ a finite dimensional manifold, union of at most countably many compact connected components, $\phi:(X, \partial X) \rightarrow(M, \partial M)$ a closed imbedding, $\tilde{\phi}_{1}, \tilde{\phi}_{2}$ two closed tubular neighborhoods which extend to the open imbeddings $\bar{\phi}_{1}, \bar{\phi}_{2},(V, \sigma)$ a collar neighborhood such that the $\tilde{\phi}_{i}$ are transversal to $(V, \sigma)$ with respect to $\bar{\phi}_{i}$ and $\bar{\phi}_{1} / \bar{\phi}_{1}^{-1}(V)=\bar{\phi}_{2} / \bar{\phi}_{1}^{-1}(V)$, then for any $0 \leqq \alpha<1$ there exists an isotopy $h_{t}:(M, \partial M) \rightarrow(M, \partial M)$ such that

(i) $h_{0}=$ id,

(ii) $h_{t} / \alpha V \cup \phi(X)=\mathrm{id} / \alpha V \cup \phi(X)$,

(iii) $h_{1} \tilde{\phi}_{1}=\tilde{\phi}_{2}$. 
The theorem is also true for any finite dimensional or $h$-manifold $X$ and closed imbedding of infinite codimension. The proof is quite the same as of Theorem 4.1 [2].

(f) A handle decomposition of $M,(M, \partial M)$, is a sequence of $0-c$ submanifolds $\left(A_{i}, \partial A_{i}\right)$ (for the case with boundary, closed submanifolds $\left(A_{i}, \partial A_{i}\right)$ with $\partial A_{i}=\partial_{0} A_{i} \cup \partial_{1} A_{i}, \partial_{0} A_{i}=\partial M$ and Int $A_{i}$ is open submanifold of Int $M$ ) with the following properties:

(i) $A_{i} \subset A_{i+1} \backslash \partial A_{i+1}$ (for the case with boundary $A_{i} \subset A_{i+1} \backslash \partial_{1} A_{i+1}$ ).

(ii) There exists a closed imbedding $\phi_{n}: \bigcup_{j=1}^{k} D_{j}^{n+1} \rightarrow\left(A^{n}, \partial A^{n}\right)$, $A^{n}=$ Int $M \backslash \operatorname{Int} A_{n}$ with $\phi_{n}\left(\cup_{j=1}^{k} D_{j}^{n+1}\right) \subset$ Int $A_{n+1} \backslash$ Int $A_{n}(\kappa$ being a positive integer or the symbol $\infty$ ) and a closed tubular neighborhood $\tilde{\phi}_{n}: \bigcup_{j=1}^{k} D_{j}^{n+1} \times D^{\infty} \rightarrow\left(A^{n}, \partial A^{n}\right)$ such that $A_{n+1}^{\prime}=A_{n} \cup \tilde{\phi}_{n}\left(\cup_{j=1}^{k} D_{j}^{n+1} \times D^{\infty}\right)$ with rounded corners, has $A_{n+1}$ as collar neighborhood.

(g) An $h$-manifold (with boundary) $M(M, \partial M)$, is called stable if it is diffeomorphic to $M \times H(M \times H, \partial M \times H)$.

Proposition 1.2. Let $M(M, \partial M)$ be an h-manifold (with boundary). The following assertions are equivalent:

(i) $M,(M, \partial M)$ is stable.

(ii) $M,(M, \partial M)$ has a handle-decomposition.

To prove the proposition, one uses the tools of $[2, \S 7]$ and Proposition 1.1.

(h) Let $(X, \partial X)$ be a finite dimensional manifold, union of at most countably many compact connected components, and $f: \partial X \rightarrow \partial M$ a closed imbedding, $(M, \partial M)$ being an $h$-manifold with boundary.

Proposition 1.3. (i) If $\bar{f}^{\prime}: X \rightarrow M$ is a continuous extension of $f: \partial X$ $\rightarrow \partial M \subset M$ there exists a closed imbedding $\bar{f}:(X, \partial X) \rightarrow(M, \partial M)$ such that $\bar{f}$ and $\bar{f}^{\prime}$ are homotopic relative to $\partial X$.

(ii) If $(V, \theta)$ is a collar neighborhood of $\partial M, f_{1}$ and $f_{2}$ are two closed imbeddings extending $f$, homotopic relative to $\partial X$ and transversal to $(V, \theta), 0 \leqq \alpha<1$, then there exists an isotopy of closed imbeddings $f_{t}$ such that $f_{t}=f_{1}=f_{2} / f_{1}^{-1}(\alpha V)$ and $f_{t}$ is transversal to $\alpha(V, \theta)$ for any $t$.

(iii) Moreover there exists an isotopy $h_{t}: M \rightarrow M$ such that $h_{0}=\mathrm{id}$ and $h_{t} \cdot f_{1}=f_{t}$.

This proposition is a very important tool in our general handle decomposition method.

(i) Let $M$ be an $h$-manifold and $\left(A_{1} \partial A_{1}\right),\left(A_{2}, \partial A_{2}\right),\left(A_{3}, \partial A_{3}\right)$ be three 0 - $c$-submanifolds such that $A_{i} \subset \operatorname{Int} A_{2}$ and $A_{1} \subset \operatorname{Int} A_{3}$. Denote by $A^{i}=M \backslash \operatorname{Int} A_{i}$.

Proposition 1.4. Suppose there exists a closed imbedding 
$f: \bigcup_{j=1}^{k}\left(D_{j}^{r_{j}}, \partial D_{j}^{\gamma_{j}}\right) \rightarrow\left(A^{1}, \partial A^{1}\right), r_{j} \leqq n$ ( $\kappa$ being a positive integer on the symbol $\infty)$ and $\tilde{f}: \mathrm{U}_{j=1}^{k} D_{j}^{r_{j}} \times D^{\infty} \rightarrow\left(A^{1}, \partial A^{1}\right)$ a closed tubular neighborhood such that $\left(A_{2}, \partial A_{2}\right)$ is a collar neighborhood of $\left(A_{2}^{\prime}, A_{2}^{\prime}\right)$ $\left(A_{2}^{\prime}=A_{1} \cup \tilde{f}\left(\bigcup_{j=1}^{k} D_{j}^{r_{j}} \times D^{\infty}\right)\right.$ with rounded corners $)$ and suppose $\pi_{i}\left(A_{3}, A_{1}\right)$ $\rightarrow \pi_{i}\left(M, A_{1}\right)$ is an epimorphism for $i \leqq n$.

Then there exists an isotopy $\phi_{i}: M \rightarrow M$ such that $\phi_{0}=\mathrm{id}, \phi_{t}=\mathrm{id} / A_{1}$ and $\phi_{1}\left(A_{2}\right) \subset$ Int $A_{3}$.

This proposition is an easy consequence of Proposition 1.4.

Using the handle decomposition one can give an easier proof of the following theorem [2].

Theorem 1.5. Any open set $U$ in the hilbert space $H$ is a stable manifold, i.e. $U$ is diffeomorphic to $U \times H$ (hence because of [1], any hilbert manifold is stable).

Proof (sKetch). Suppose $0 \in H$ contained in $U, e_{1}, \cdots, e_{n}$, an orthonormal basis of $H$ and $R^{k}$ the finite dimensional subspaces generated by $e_{1}, \cdots, e_{k} . U_{k}=U \cap R^{k}$ are closed submanifolds of $U$ of finite dimension. One can find a $C^{\infty}$-function $\rho: U \rightarrow R_{+}$such that for any point $x$, the ball centered in $x$, of radius $\rho(x)$, is contained in $U$. For any $\alpha<1$ one defines $B_{n, \alpha}$ the closed tubular neighborhood of $U_{n}$ of radius $\alpha \rho(x)$ which is a stable manifold with boundary, hence $B_{n, \alpha}=B_{n, \alpha} \times H$. Denote by $V_{1}, \cdots V_{k}, \cdots$ the manifolds $B_{1, \rho / 2} \ldots$; $B_{k},\left(1 / 2+\cdots 1 / 2^{k}\right) \rho$, and remark $V_{k} \subset$ Int $V_{k+1}$ and $U_{k}$ Int $V_{k}=U$. Because $V_{k}$ is stable, $V_{k+1}$ is a $0-c$-submanifold of $U$ which can be obtained adding handles of dimension $\leqq k+1$ to $V_{k}$. Combining with Proposition 1.4 we can find a sequence of hilbert manifolds $L_{k}, L_{k}$ openly imbedded in $L_{k+1}$, and a sequence of diffeomorphisms $h_{k}$, such that the diagram is commutative:

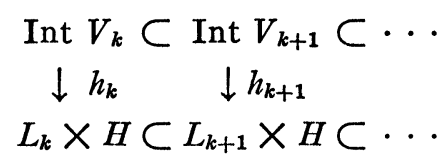

THEOREM 1.6. If $(M, \partial M)$ is a hilbert manifold with boundary whose boundary has the homotopy type of a finite dimensional CW-complex, $(M, \partial M)$ is stable.

This theorem is a consequence of $1.2,1.4$, and 1.5.

(j) Lemma 1.7. There exists a diffeomorphism $\nu: H \rightarrow H \times H$ such that $\nu\left(D^{\infty}\right)=D^{\infty} \times H$.

(Essentially proved in $[2, \S 2]$.) 
(k) A diffeomorphism $l: M \times H \rightarrow M \times H$ is called stable if $l=t \times$ id where $t$ is a diffeomorphism of $M$.

THEOREM 1.8. Any diffeomorphism $\phi: M \times H \rightarrow M \times H$ is isotopic to a stable diffeomorphism.

This theorem is the crucial step of our paper. It is an inductive consequence of Lemma 1.9 combined with Proposition 1.2.

Let $M$ be a hilbert manifold $\left\{\left(A_{n}, \partial A_{n}\right)_{n=1,2}, \ldots\left(\phi_{n}, \tilde{\phi}_{n}\right)_{n=1,2, \ldots}\right.$, a handle decomposition and $l: M \times H \rightarrow M \times H$ be a diffeomorphism, $l(x, v)=\left(l^{1}(x, v), l^{2}(x, v)\right) \in M \times H$.

LEMma 1.9. Suppose $l$ verifies the following conditions:

$\left(\mathrm{i}_{n}\right) l^{1}(x, v)=l^{1}(x, 0), l^{2}(x, v)=v$ for any $x^{\prime} \in A_{n}, v \in H$ where ( $\left.A_{n}, \partial^{\prime} A_{n}\right)$ is a collar neighborhood of $\left(A_{n}, \partial A_{n}\right)$.

(ii $\left.{ }_{n}\right) l^{1}\left(A_{k} \times 0\right) \subset$ Int $A_{k} \times 0$ for any $k \leqq n-1$, and $l^{1}\left({ }^{\prime} A_{n} \times 0\right) \subset A_{n} \times 0$.

(iii $\left.{ }_{n}\right) l^{1}\left(A_{k} \times 0\right) \supset A_{k-1} \times 0$ for any $k \leqq n$. Then, given $\left({ }^{\prime} A_{n+1}, \partial^{\prime} A_{n+1}\right)$ a collar neighborhood of $\left(A_{n+1}, \partial A_{n+1}\right)$ there exists an isotopy $l_{t}$ such that:

(1) $l_{t}=l$ on $A_{n-1} \times H, l_{0}=l$.

(2) $\left(\mathrm{i}_{n+1}\right),\left(\mathrm{ii}_{n+1}\right)$, and $\left(\mathrm{iii}_{n+1}\right)$ are verified.

(3) $l_{t}=l$ for $0 \leqq t \leqq 1 / 3, l_{t}=l_{1}$ for $2 / 3 \leqq t \leqq 1$.

To get (1) we need the remarks (b), (c), (d), Propositions 1.1, 1.3, and Lemma 1.7. To get (2) we need more, Proposition 1.5.

(2) (a) Proposition 2.1. Let $l: M \times H \rightarrow M \times H$ be a diffeomorphism such that $l / M \times 0=\mathrm{id}$. Then $l$ is isotopic to the identity.

The proof uses the contractibility of the general linear group of $H$, and:

Hirsch's Lemma (3.1, [2]). Let $[b, c]$ and $[a, e]$ be two closed intervals with $a<b<c<e$ and $F: A=[a, e] \rightarrow B \times[a, e]$ be an isotopy of closed imbeddings (a level preserving closed imbedding) between $A, B$ hilbert manifolds, with parameter $t \in[a, e]$.

If $F_{1}: A \times[a, e] \rightarrow B$ is an imbedding where $F(x, t)=\left(F_{1}(x, t), t\right)$, then there exists an ambient isotopy $G: B \times[b, c] \rightarrow B \times[b, c]$ (of diffeomorphisms of $B)$ such that $G\left(F_{1}(x, t), t\right)=F(x, t)$ for $(x, t) \in A \times[b, c]$.

THEOREM 2.2. Let $q: M \rightarrow M$ be a diffeomorphism homotopic to the identity; then $q \times \mathrm{id}: M \times H \rightarrow M \times H$ is isotopic to the identity.

The proof is a consequence of Proposition 2.1, of the Hirsch lemma, and of the close imbeddability of $M$ in $S^{\infty}$ with infinite dimensional normal bundle. 
(b) THEOREM 2.3. Two homotopic diffeomorphisms $h_{i}: M \rightarrow M$, $i=1,2$ are isotopic.

Proof. Of course, it is sufficient to prove our assertion in the case $h_{0}=\mathrm{id}$.

According to Theorem 1.5 we can suppose $M=M \times H$. Then the theorem can be obtained combining Theorems 1.8 and 2.2.

REMARK. Theorem 2.3 is not true for Banach manifolds, not even for parallelizable Banach manifolds. For instance, for the manifold $l_{2} \oplus c_{0}$, considered as $l_{2} \oplus c_{0}$-manifold Douady constructed two linear isomorphisms which are not isotopic by linear isomorphism. Supposing these were isotopic by diffeomorphisms, a standard trick (taking Jacobians) would give an isotopy by linear isomorphisms.

Note ADDED IN PROOF. Using different methods D. Elworthy can also prove Theorem 2.3.

\section{BIBLIOGRAPHY}

1. J. Eells and D. Elworthy, Open imbedding for banach manifolds (preprint) (to appear).

2. D. Burghelea and N. Kuiper, Hilbert manifolds, Ann. of Math. (2) 90 (1969), 379-417.

3. S. Lang, Introduction to differentiable manifolds, Interscience, New York, 1962. MR 27 \#5192.

4. N. Moulis, Sur les variétés hilbertiennes et les fonctions nondégénérées, Indag. Math. 30 (1968), 497-511.

Mathematical Institute of the Rumanian Academy, Bucharest, Rumania and Institute for Advanced Study, Princeton, New Jersey 08540 